\title{
Four Ways to Compute the Inverse of the Complete Elliptic Integral of the First Kind
}

\author{
John P. Boyd \\ Department of Atmospheric, Oceanic \& Space Science \\ University of Michigan, 2455 Hayward Avenue, Ann Arbor MI 48109 \\ jpboyd@umich.edu
}

\begin{abstract}
The complete elliptic integral of the first kind arises in many applications. This article furnishes four different ways to compute the inverse of the elliptic integral. One motive for this study is simply that the author needed to compute the inverse integral for an application. Another is to develop a case study comparing different options for solving transcendental equations like those in the author's book [11]. A third is to develop analytical approximations, more useful to theorists than mere numbers. A fourth is to provide robust "black box" software for computing this function. The first solution strategy is "polynomialization" which replaces the elliptic integral by an exponentially convergent series of Chebyshev polynomials. The transcendental equation becomes a polynomial equation which is easily solved by finding the eigenvalues of the Chebyshev companion matrix. (The numerically ill-conditioned step of converting from the Chebyshev to monomial basis is never necessary). The second approximation is a regular perturbation series, accurate where the modulus is small. The third is a power-and-exponential series that converges over the entire range parameter range, albeit only sub-exponentially in the limit of zero modulus. Lastly, Newton's iteration is promoted from a local iteration to a global method by a Never-Failing Newton's Iteration (NFNI) in the form of the exponential of the ratio of a linear function divided by another linear polynomial. A short Matlab implementation is provided, easily translatable into other languages. The Matlab/Newton code is recommended for numerical purposes. The other methods are presented because (i) all are broadly applicable strategies useful for other rootfinding and inversion problems (ii) series and substitutions are often much more useful to theorists than numerical software and (iii) the Never-Failing Newton's Iteration was discovered only after a great deal of messing about with power series, inverse power series and so on.
\end{abstract}

Keywords: pseudospectral; Chebyshev polynomials ; elliptic integral; inverse elliptic integral

Preprint submitted to Elsevier

February 2, 2015

(C) 2015. This manuscript version is made available under the Elsevier user license http://www.elsevier.com/open-access/userlicense/1.0/ 


\section{Introduction}

The complete elliptic integral arises in a thousand applications, and various approximations and series for it can be found in a variety of sources such as Chapter 18 of the NIST Digital Library of Functions [31]. However, no simple form for the inverse is listed in any of these references. The complete elliptic integral of the first kind can be written in multiple forms.

$$
\begin{aligned}
K(m) & =\int_{0}^{\pi / 2} \frac{d t}{\sqrt{1-t^{2}} \sqrt{1-m t^{2}}} \\
& =\frac{\pi}{2} \theta_{3}^{2}(0 ; \tau), \quad m=\frac{\theta_{2}^{4}(0 ; \tau)}{\theta_{3}^{4}(0 ; \tau)} \\
& =\operatorname{arcsn}(\pi / 2 ; m)
\end{aligned}
$$

where $\theta_{3}$ is the standard Jacobian theta function and arcsn is the inverse of the elliptic sine [31] The inverse problem is to compute $m(\lambda)$ that solves

$$
K(m)-\lambda=0, \quad \lambda \in[\pi / 2, \infty], m \in[0,1]
$$

Unfortunately, none of these forms is much use in inverting the integral. The inverse of the Jacobian elliptic function looks promising, but this and the incomplete elliptic integral are both bivariate functions where one argument is usually a coordinate in applications and the other is the elliptic modulus $m$, which is typically a parameter. The elliptic sine is the inverse of the incomplete elliptic integral with respect to the coordinate. The inverse of $K(m)$ is the inverse with respect to the parameter.

Fukushima [26] catalogs some applications for $K^{-1}(m)$. Our motive was two different applications: (i) nonlinear wave theory where $K(m)$ is the period for traveling wave solutions to classical nonlinear wave equations and (ii) construction of new, more uniform grids for Chebyshev spectral methods by replacing circular functions and their inverses by elliptic functions and their inverses.

Amore [2] shows that the singularity of the deflection angle of a light ray in the Schwarzschild metric is given by the inverse of a function derived from the elliptic integral, though not the elliptic integal itself. In other articles, Amore and Fernandez $[4,3]$ have shown that often the best perturbation expansion for a function like $K(m)$ is a perturbation series for the inverse. Although they never treat $K^{-1}(m)$ explicitly, but only closely related functions, their work emphasizes that it is difficult to fully understand and optimally analyze a function without its inverse.

However, applications are only part of our motivation. Another goal to use this example to illustrate a quartet of rather general strategies for function inversion. The inverse elliptic integral is a good exemplar partly because it does have practical applications and partly because it has a rich history more than two centuries old. This example is also good because it is hard: the range of the argument $\lambda$ of the inverse is infinite and the elliptic integral is logarithmically singular as $m \rightarrow 1$. 
Nevertheless, three of our four methods are global, converging over the entire branch. The elliptic integral is a good illustration of the strengths and drawbacks of the numerical and analytic inversion methods analyzed here.

Polynomialization is an improvement on the widely-used Chebyshev-proxy rootfinder in which real roots on an interval are calculated by (i) expanding $f(x, \lambda)$ as a Chebyshev series in $x$ on the interval and then finding the roots of this polynomial proxy by computing the eigenvalues of the Chebyshev companion matrix whose elements are trivial functions of the Chebyshev coefficients [10]. Usually the proxy must be computed anew for each $\lambda$. Here, we shall obtain a single proxy which is valid for the entire range, even though that domain in $\lambda$ is unbounded.

We shall discuss each of the four inversion methods in its own section and then make some summary remarks at the end. This work is a further case study illustrating the methodologies for inversion and rootfinding discussed in the author's book [7].

\section{Polynomialization of the Complete Elliptic Integral of the First Kind}

It is convenient to introduce a "complementary modulus" $x$ defined by

$$
x \equiv 1-m
$$

because the integral is singular as $m \rightarrow 0$, which is equivalent to $x \rightarrow 0$. (In the elliptic function literature, this quantity is usually denoted $m^{\prime}$, but we have chosen $x$ to emphasize that this parameter is the unknown in the transcendental equation solved here.) Polynomialization over the entire interval $m \in[0,1]$ is hard because for small $x$

$$
K(m)=K(1-x) \sim\left(-\frac{1}{2}-\frac{1}{8} x\right) \log (x)+\log (4)+\left(\frac{1}{2} \log (2)-\frac{1}{4}\right) x(6)
$$

To deal with this, we use a logarithmic transformation from $x \in[0,1]$ to $y \in$ $[0, \infty]$ :

$$
x=\exp (-y)
$$

We then apply the inverse map used to convert the rational Chebyshev functions for the semi-infinite interval into Chebyshev polynomials on $z \in[-1,1][5]$ :

$$
y=L \frac{1+z}{1-z}
$$

which implies

$$
x=\exp \left(-L \frac{1+z}{1-z}\right)
$$


The elliptic integral can be written without approximation as

$$
K(m)=K(1-x) \sim Q(x) \log (x)+P(x)
$$

where $P$ and $Q$ have ordinary convergent power series. Inserting the map gives

$$
\begin{aligned}
K(m)=K(1-x) & \sim Q(x) \log \left(\exp \left(-L \frac{1+z}{1-z}\right)\right)+P(x) \\
& =Q(x[z])\left(-L \frac{1+z}{1-z}\right)+P(x[z])
\end{aligned}
$$

$K$ is thus unbounded as $z \rightarrow 1$, and therefore does not have a bounded TL series. The singular term is $-Q(x[z]) L(1+z) /(1-z)$, which cannot be subtracted unless one writes a new special function routine to evaluate $Q$.

So, we shall instead solve $K(m=1-x)=\lambda$ by solving

$$
K(m=1-x)(1-z)=\lambda(1-z)
$$

We expand $K(m=1-x[z])(1-z)$ as a Chebyshev polynomial series in $z$ up to and including degree $N, K_{N}(z)$, and solve the polynomial equation

$$
K_{N}(z)-\lambda(1-z)=0
$$

The mechanics of solving polynomial equations in Chebyshev form is described in the appendix. Denote the unique zero of $K_{N}(z)-\lambda(1-z)$ on $z \in[-1,1]$ by $z_{*}$. The $x$ value is

$$
x=\exp \left(-L \frac{1+z_{*}}{1-z_{*}}\right)
$$

The elliptic modulus $m(\lambda)$, the inverse of the elliptic integral, is

$$
m=1-\exp \left(-L \frac{1+z_{*}}{1-z_{*}}\right)
$$

One might expect loss of accuracy for $z \approx 1$ where $x \approx 0$ and $m \approx 1$. However, tests show little error is this limit.

Approximation of $K(1-z)$ to near Matlab/IEEE double precision, $2 \times 10^{-16}$, can be obtained from 50 terms in the Chebyshev series and $L=8$. Using The Chebyshev series in $z$ can also be interpreted as a series of rational Chebyshev functions $\mathrm{TL}_{n}(y[\lambda])$. The usual rate of convergence for rational Chebyshev series is exponential but subgeometric [6]; Fig. 1 suggests that this expectation is true. However, multiple precision was needed here to observe that the coefficients do not asymptote to a straight line (falling as $\exp (-q n)$, a geometric rate of convergence), but rather show a slight upward curvature.

It is necessary to switch to the asymptotic expansion for $|x| \leq 10^{-6}$ to avoid overflow errors in 16 decimal digit precision. 


$$
\begin{aligned}
K(1-x[z])(1-z) \sim & (1-z)\left\{\log (4)+\left(\frac{1}{2} \log (2)-\frac{1}{4}\right) x[z]+\left(\frac{9}{32} \log (2)-\frac{21}{28}\right) x[z]^{2}\right\} \\
& -L(1+z)\left(-\frac{1}{2}-\frac{1}{8} x[z]-\frac{9}{128} x[z]^{2}\right), \quad|x| \ll 1[m \approx 1]
\end{aligned}
$$




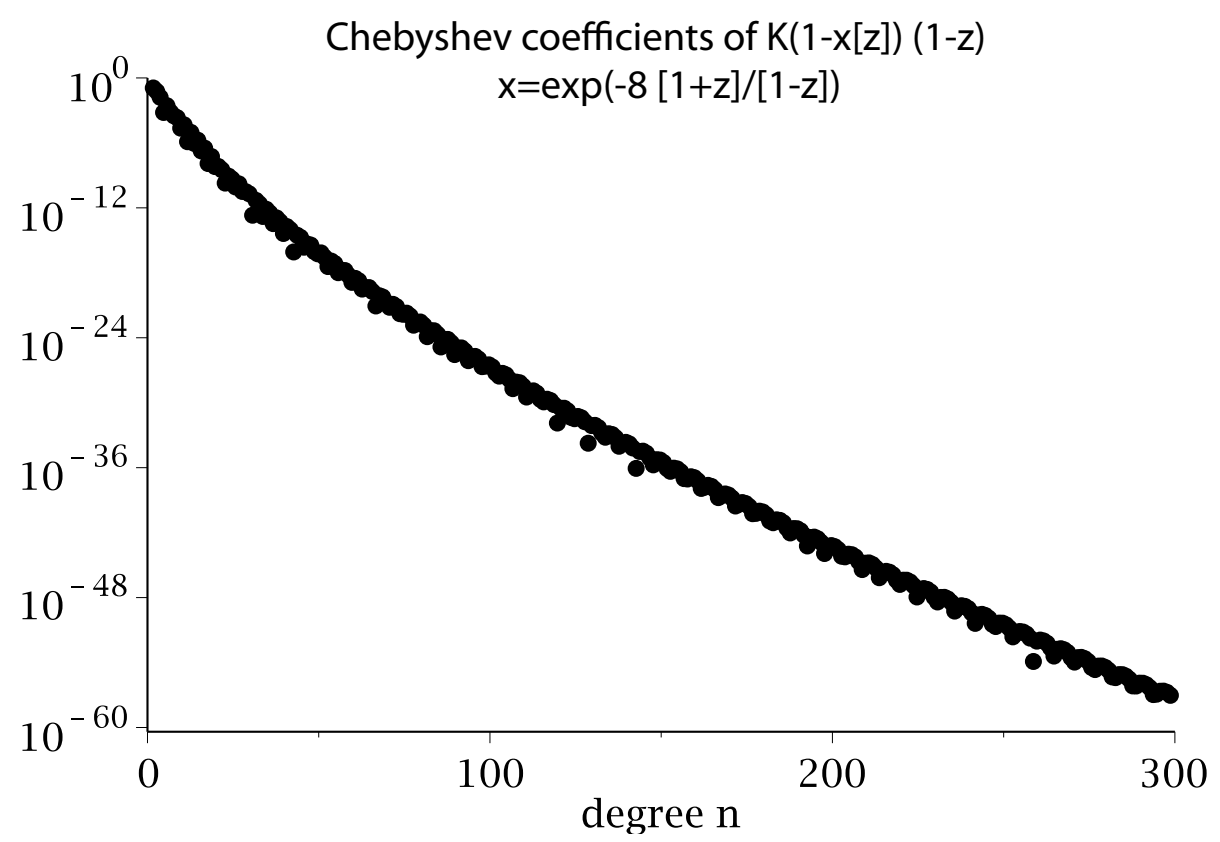

Figure 1: Coefficients of the expansion of $K(1-\exp (-L(1+z) /(1-z)))(1-z)$ where $K(m)$ is the complete elliptic integral of the first kind and $L=8$. The slight upward curvature on this log-linear plot shows that the rate of convergence is "subgeometric". Typically, the coefficients are $O\left(\exp \left(-q n^{r}\right)\right.$ where $q$ and $r$ are positive constants. By definition, the exponent of subgeometric convergence $r$ is less than one but greater than zero. Because the curve bends upward so gently, multiple precision arithmetic was applied. 


\section{Power Series for Small Modulus}

When $m$ is small, $K(m)-\lambda$ has a rapidly convergent power series. Because $K(0)=\pi / 2$, the small parameter for the approximation of the inverse, $K^{-1}(\lambda)$, is

$$
\epsilon \equiv \lambda-\pi / 2
$$

Replacing $\lambda$ by $\epsilon+\pi / 2$, the series for the function $f(m, \lambda) \equiv K(m)-\lambda$ whose root defines $K^{-1}(\lambda)$ is

$$
\begin{aligned}
K(m)-\lambda= & -\epsilon+\frac{1}{8} \pi m+\frac{9}{128} \pi m^{2}+\frac{25}{512} \pi m^{3}+\frac{1225}{32768} \pi m^{4}+\frac{3969}{131072} \pi m^{5} \\
& +\frac{53361}{2097152} \pi m^{6}+\frac{184041}{8388608} \pi m^{7}+\frac{41409225}{2147483648} \pi m^{8}+\ldots \quad \text { (21) }
\end{aligned}
$$

The inverse series is derived by substituting the formal series

$$
m=\sum_{j=1}^{N} b_{j} \epsilon^{j}
$$

into the power series for $K-\lambda$, up to and including $O\left(m^{N}\right)$ and collecting powers of $\epsilon$. To fourth order, the result is

$$
\begin{aligned}
K-\lambda= & \sum_{j=1}^{N} r_{j}\left(b_{1}, \ldots, b_{j}\right) \epsilon^{j} \\
= & \left(-1+\frac{\pi}{8} b_{1}\right) \epsilon+\left(\frac{\pi}{8} b_{2}+\frac{9}{128} \pi b_{1}{ }^{2}\right) \epsilon^{2}+\left(\frac{9}{64} \pi b_{1} b_{2}+\frac{25}{512} \pi b_{1}{ }^{3}+\frac{\pi}{8} b_{3}\right) \epsilon^{3} \\
& +\left(\frac{9}{128} \pi\left(b_{2}{ }^{2}+2 b_{3} b_{1}\right)+\frac{1225}{32768} \pi b_{1}{ }^{4}+\frac{75}{512} \pi b_{1}{ }^{2} b_{2}+\frac{\pi}{8} b_{4}\right) \epsilon^{4}+\ldots
\end{aligned}
$$

Equating the $O(\epsilon)$ term to zero determines $b_{1}$, solving $r_{1}\left(b_{1}, b_{2}\right)=0$ yields $b_{2}$ and in general $r_{j}=0$ determines $b_{j}$ to all orders.

The first few coefficients are given in the table as $\tilde{b}_{n} \equiv(\pi / 2)^{n} b_{n} / n^{2}$. The signs are strictly alternating, which is the sign of a singularity on the negative real axis [32]. ${ }^{1}$ It is often possible to estimate to estimate the radius of convergence; if $b_{j} \sim A \rho^{-n}\left(n^{k}+B n^{k-1}\right)$, then

$$
\exp \left(\log \left(\left|a_{n}\right|\right) /(-n)\right) \sim \rho-\rho k \frac{\log (n)}{n}-\rho \log (A) \frac{1}{n}+O\left(\frac{1}{n^{2}}\right)
$$

Curve fitting of coefficients 70 to 100 yields $\rho \approx 1.5703$, which differs negligibly from $\pi / 2=1.5708$. The radius of convergence is almost certainly $\pi / 2$ with the singularity at $\epsilon=-\pi / 2$, the point where $K^{-1}(0)$ is singular. The domain of convergence in modulus is $m \in[0,0.9691]$.

\footnotetext{
${ }^{1}$ This accords with the intuition that the singularity is where the series converges most slowly, which for an alternating power series is the negative real axis.
} 
Table 1: Scaled coefficients $\tilde{b}_{n} \equiv(\pi / 2)^{n} b_{n} / n^{2}$ for the $\epsilon$-power series coefficients for $K^{-1}(\pi / 2+$ $\epsilon)$.

\begin{tabular}{|c|c|l|}
\hline$n$ & $\hat{b}_{n}$ & $\hat{b}_{n}$ [floating point] \\
\hline 1 & 4 & 4. \\
2 & $-9 / 4$ & -2.25 \\
3 & $\frac{31}{18}$ & 1.7222 \\
4 & $-\frac{185}{128}$ & -1.4453 \\
5 & $\frac{507}{400}$ & 1.2675 \\
6 & $-\frac{5257}{4608}$ & -1.1408 \\
7 & $\frac{6551}{6272}$ & 1.0445 \\
8 & $-\frac{126855}{131072}$ & -.9678 \\
9 & $\frac{300215}{33176}$ & .9049 \\
10 & $-\frac{139579}{163840}$ & -.8519 \\
\hline
\end{tabular}

The radius of convergence can be extended by Padé approximants, Euler sum acceleration and so on. Because the series is strictly alternating, the sum is particularly well-suited for Euler acceleration [32, 29, 8, 12]. Table 2 shows that the radius of convergence of the power series $((\lambda-\pi / 2)<\pi / 2$ is nearly tripled to about $(\lambda-\pi / 2)<2.8(\pi / 2)$, but the asymptotic series is the better way.

\section{Asymptotic Power-and-Exponential Series for the Inverse of the Complete Elliptic Integral of the First Kind}

In this section, we derive an asymptotic series for the inverse $x(\lambda)$ of the function $K(m)$ where the modulus of the elliptic function is $m=1-x$. It is convenient to work with $x$ instead of $m$ because the logarithmic singularity of the elliptic integral is at small $x$. (Note that elliptic functions are often evaluated using the alternative "modulus", $k=\sqrt{m}$, as is true in the computer language Maple.)

The first step is to write the first terms of the expansion of the elliptic integral for small $x$, as can be looked up in any reference on special functions:

$$
K(1-x) \sim-(1 / 2) \log (x)+\log (4)
$$

Inserting this in the equation $K=\lambda$ gives something simple enough to be explicitly solved for the lowest order approximation:

$$
x \sim 16 \exp (-2 \lambda), \quad \lambda>>1
$$

To proceed further, we define the lowest order approximation to be the perturbation parameter

$$
\epsilon=16 \exp (-2 \lambda)
$$


Table 2: Errors in the Power Series, Euler-Accelerated Power Series and Asymptotic Expan-

sion

\begin{tabular}{|c|c|c|c|c|}
\hline$\lambda /(\pi / 2)-1$ & $\mathrm{~N}$ & N-term Eulerized error & N-term Power Series & 4th Order Asymptotic \\
\hline 0 & any & 0 & 0 & 0.118 \\
\hline 1 & 30 & $5.0 \mathrm{E}-10$ & 8.2 & - \\
1 & 60 & $4.9 \mathrm{E}-18$ & 0.90 & - \\
1 & 100 & $1.2 \mathrm{E}-27$ & 0.016 & - \\
1 & 125 & $1.2 \mathrm{E}-33$ & 0.0093 & 0.0000040 \\
\hline 2 & 30 & 0.000011 & $2.9 \mathrm{E} 11$ & - \\
2 & 60 & $1.6 \mathrm{E}-12$ & $3.6 \mathrm{E} 19$ & - \\
2 & 100 & $3.6 \mathrm{E}-19$ & $1.0 \mathrm{E} 31$ & $5.9 \mathrm{E}-11$ \\
2 & 125 & $1.2 \mathrm{E}-23$ & $1.2 \mathrm{E} 38$ & - \\
2.5 & 30 & 0.10 & $7.3 \mathrm{E} 13$ & - \\
2.5 & 60 & 0.000046 & $4.7 \mathrm{E} 25$ & - \\
2.5 & 100 & $9.1 \mathrm{E}-10$ & $1.5 \mathrm{E} 41$ & $1.9 \mathrm{E}-13$ \\
2.5 & 125 & $9.1 \mathrm{E}-13$ & $6.5 \mathrm{E} 50$ & - \\
\hline 3 & 30 & 524 & $2.2 \mathrm{E} 16$ & - \\
3 & 60 & 1326 & $4.3 \mathrm{E} 30$ & - \\
3 & 125 & 3457 & $1.3 \mathrm{E} 61$ & $5.6 \mathrm{E}-16$ \\
\hline 3 & 150 & 4369 & $5.9 \mathrm{E} 72$ & \\
\hline
\end{tabular}

and expand

$$
x \sim \epsilon+\sum_{n=2}^{\infty} b_{n} \epsilon^{n}
$$

The next step is to write down the small- $x$ series for $K$ to higher order - note that $x^{n}$ will be $O\left(\epsilon^{n}\right)$ - but there is a complication. Although $\exp (-2 \lambda)$ is very small, and is proportional to the perturbation parameter, the logarithm of this, $\log (\exp (-2 \lambda))=-2 \lambda$, which is large. To get the correct order, we must help our computer algebra system by writing

$$
\begin{aligned}
\log \left(\epsilon+\sum_{n=2}^{\infty} b_{n} \epsilon^{n}\right) & =\log \left(\epsilon\left[1+\sum_{n=2}^{\infty} b_{n} \epsilon^{n-1}\right]\right) \\
& =\log (\epsilon)+\log \left(1+\sum_{n=2}^{\infty} b_{n} \epsilon^{n-1}\right) \\
& =\log (16)-2 \lambda+\log \left(1+\sum_{n=2}^{\infty} b_{n} \epsilon^{n-1}\right)
\end{aligned}
$$

The terms are now properly ordered and a few lines of Maple does the rest (Table 3).

The first few orders are, recalling $\epsilon=16 \exp (-2 \lambda)$,

$$
x \sim \epsilon+\left(\frac{\lambda-1}{2}\right) \epsilon^{2}+\left(\frac{3}{8} \lambda^{2}-\frac{19}{32} \lambda+\frac{11}{64}\right) \epsilon^{3}
$$


Table 3: Maple Code for the Large $\lambda$ Expansion of the Inverse of the Complete Elliptic Integral of the First Kind

restart: N_in_inverse:= 5; \# Numbers of terms sought for x(lambda);

Ntruncation:=N_in_inverse +3 ; \# number of terms kept in the small $x$-series; restof $\mathrm{x}:=0$; for $\mathrm{j}$ from 2 to N_in_inverse do restof $\mathrm{x}:=$ restof $\mathrm{x}+\mathrm{b}[\mathrm{j}]^{*}$ epsilon $* * \mathrm{j}$; od: \# First step: expand $\mathrm{f}(\mathrm{x}$, lambda) for small $\mathrm{x}$; $\mathrm{f}:=$ EllipticK(sqrt(1-x)) - lambda; fs:= convert(series(f,x,Ntruncation),polynom); \# special treatment for the logarithm: $\log (\mathrm{x})=\log ($ epsilon $) * \log (1+$ restofx/epsilon $)$; \# explicitly evaluate $\log ($ epsilon $)=\log (16)-2 * \operatorname{lambda}+\log (1+$ restofx/epsilon $)$; fss: $=\operatorname{subs}(\log (\mathrm{x})=\log (16)-2 * \operatorname{lambda}+\log (1+\operatorname{restofx} /$ epsilon $)$, fs $)$;

\# Third step: substitute into the small-x series and expand in epsilon;

$\mathrm{x}:=$ epsilon + restofx; resid:=series(fss,epsilon,Ntruncation);

for $\mathrm{j}$ from 2 to N_in_inverse do $\mathrm{r}[\mathrm{j}-1]$ := coeff(resid,epsilon, $\mathrm{j}-1$ );

$\mathrm{b}[\mathrm{j}]:=\operatorname{solve}(\mathrm{r}[\mathrm{j}-1], \mathrm{b}[\mathrm{j}])$; od:

print $\left({ }^{6}\right.$ asymptotic series, $\mathrm{x}($ lambda $)={ }^{6}, \operatorname{collect}(\operatorname{simplify}(\mathrm{x})$, epsilon $\left.)\right)$;

$$
+\left(\frac{1}{3} \lambda^{3}-\frac{11}{16} \lambda^{2}+\frac{47}{128} \lambda-\frac{3}{64}\right) \epsilon^{4}, \quad \lambda>>1
$$

Note that the coefficient of $\epsilon^{n}$ is a polynomial of degree $(n-1)$ in $\lambda$. Although the powers of $\lambda$ cause no complications, this asymptotic expansion is a powerand-exponential series in $\lambda$ (or a log-and-power series in $\epsilon$ ) and not a pure power series.

The fourth order is accurate to Matlab machine precision for $\lambda>6$, the third order for $\lambda>6.5$, the second order for $\lambda>8.0$ and the lowest order for $\lambda>10.8$ (Fig. 3).

At the endpoint $\lambda=\pi / 2$, the tenth, twentieth and thirtieth orders give $m=0.03579,0.01786$ and 0.01191, apparently converging slowly to the true limiting value of zero. This global convergence for a power-and-exponential series is remarkable.

The approximation at $N$-th order in $\epsilon$ is a polynomial of degree $(N-1)$. The $N$-th order power series for small $\lambda-\pi / 2$ contains only $N$ terms whereas the $N$-th order for the large- $\lambda$ series contains roughly $O\left(N^{2} / 2\right)$ terms. We could easily calculate the small $(\lambda-\pi / 2)$ series to 150 terms whereas attempting to go above $N=36$ for the asymptotic series crashed our laptop. 


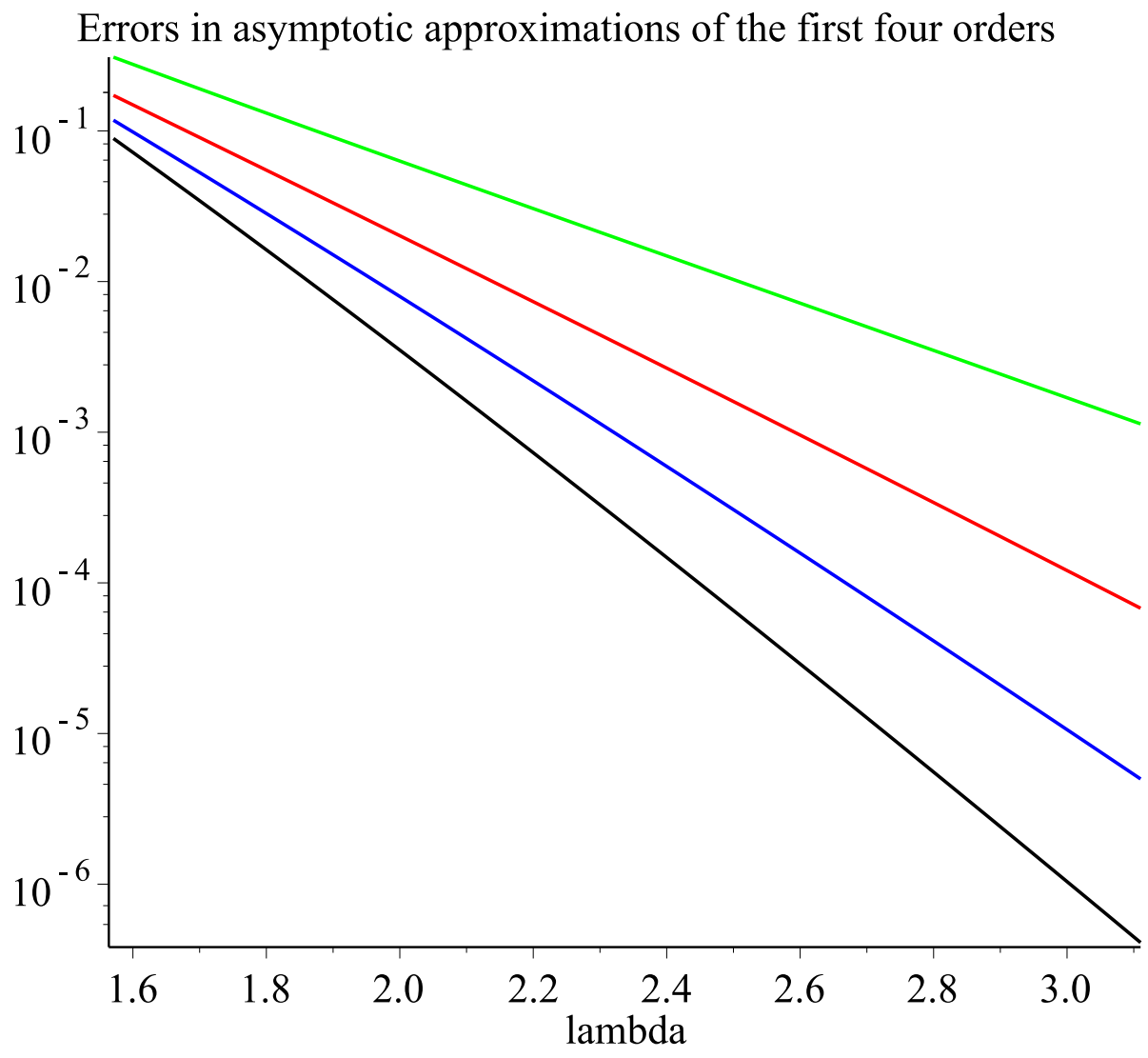

Figure 2: Errors in the asymptotic approximations of the first four orders for large $\lambda$, shown on the interval $\lambda \in[\pi / 2, \pi]$ where the small $\lambda$ series converges. The $O(\exp (-8 \lambda)$ expansion predicts $m=0.089$ at $\lambda=\pi / 2$ where the exact answer is $m=0$. However, $K(0.89)=$ $1.023(\pi / 2)$, so the error is small in a backwards error sense. 


\section{Never-Failing Newton Initialization}

Newton's iteration is powerful if and only if it can be provided with a NeverFailing Newton Initialization (NFNI). We can generate one by using the perturbative results derived below. It is convenient to introduce

$$
\bar{\lambda} \equiv \lambda-\pi / 2 \quad \leftrightarrow \lambda=\bar{\lambda}+\pi / 2
$$

Then the perturbative constraints to low order are, using the complementary modulus $x \equiv 1-m$,

$$
\begin{aligned}
& x \sim 1-\frac{8}{\pi} \bar{\lambda}, \quad \bar{\lambda}<<1 \\
& x \sim 16 \exp (-\pi) \exp (-2 \bar{\lambda}), \quad \bar{\lambda}>>1
\end{aligned}
$$

Mimicking the exponentials found useful in polynomialization, write

$$
x \sim \exp (H)
$$

where

$$
\begin{gathered}
H \equiv \bar{\lambda} \frac{d_{0}+d_{1} \bar{\lambda}}{1+d_{2} \bar{\lambda}} \\
x=\exp (H(\bar{\lambda}) \\
\approx \exp (H(0))+\exp (H(0)) H^{\prime}(0) \bar{\lambda}
\end{gathered}
$$

Noting that $x=\exp \left(H(\bar{\lambda}) \approx \exp (H(0))+\exp (H(0)) H^{\prime}(0) \bar{\lambda}\right.$, the small $\bar{\lambda}$ constraints require

$$
\begin{aligned}
H(0) & =1 \\
H^{\prime}(0) & =-\frac{8}{\pi}
\end{aligned}
$$

For large $\bar{\lambda}$

$$
H \sim-2 \bar{\lambda}+\{\log (16)-\pi\}+O(1 / \bar{\lambda}))
$$

Matching the constraints is an exercise in linear algebra that yields

$$
d_{0}=\frac{8}{\pi} \quad d_{1}=4 \frac{4-\pi}{\pi(\pi-4 \ln (2))} \quad d_{2}=d_{1} / 2
$$

The maximum peak relative error is 0.081 at $x=0.0223$, which is unimpressive in of itself. However, this is only the start of the iteration; careful numerical experiments show that is a Never-Failing Newton Initialization.

The Newton iteration is

$m^{n+1}=m^{n}-K\left(m^{n}-\lambda\right) /\left\{\frac{E\left(m^{n}\right)-(1-m) K\left(m^{n}\right)}{2 m(1-m)}\right\}, \quad m=0,1, \ldots$ 
Table 4: A Matlab function to compute the inverse $m(\lambda)$ of the Complete Elliptic Integral of the First Kind, $K(m)$

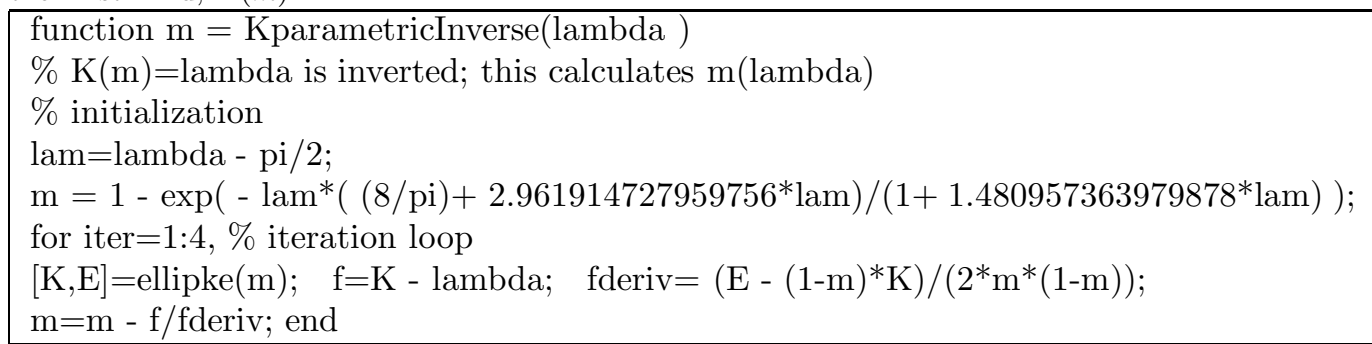

where as derived above the initialization is

$$
m^{0}=1-\exp \left(-[\lambda-\pi / 2] \frac{(8 / \pi)+(\lambda-\pi / 2) 2.961914727959756}{1+(\lambda-\pi / 2) 1.480957363979878}\right)
$$

Table 4 is a Matlab implementation. Only four Newton iterations are employed because further iterations produced no improvement.

Fig.. 4 shows that the final Newton iteration is close to machine pecision for $\lambda<5$, but then rises as roughly $10^{-17} /(1-m)$. Since the error is tiny except for extremely small $x$, no effort has been made to reduce it.

\section{Summary}

Although articles on computing $K(m)$ itself are extensive $[13,14,15,17,18$, $19,20,21,22,23,24,25,28,33]$ and various highly accurate approximations are given in reference books such as $[1,31,30,16]$, works on inverting elliptic integrals are rare $[9,24,27]$. The only other work on inverting the complete elliptic integral of the first kind is Fukushima [26], which was published after this article was almost complete. His objective was to produce a library routine optimized for speed and reliability. His procedure splits the semi-infinite domain into eight subintervals and applies a separate Chebyshev expansion to approximate $f$ piecewise over the entire domain.

We make no claim that any of our four inversion strategies can improve upon Fukushima's for speed. However, solving a univariate transcendental equation requires a fraction of a microsecond on a contemporary computer. For numerical purposes, we recommend Newton's iteration with the Never-Failing Initialization given in the pevious section. The Matlab, which has only seven executable statements, is easily translated to other programming languages.

We presented three other inversion methods in furtherance of two other goals. One is to provide expansions useful to theorists. The other is to use this inversion problem as a case study of equation-solving, a sort of post-publication annex to the case studies in the author's book [11].

Polynomial approximations are usually restricted to a finite interval, but here we map this interval to the canonical $z \in[-1,1]$ using the same change of 
coordinate as is used to generate the rational Chebyshev functions $[5,6]$. It is not necessary to compute a different polynomial in $z$ for each different value of the parameter $\lambda$, as in the usual Chebyshev proxy algorithm [10]; one expansion works for the entire branch.

The power series in the small parameter $\epsilon=\lambda-\pi / 2$ only converges as far as $\lambda=\pi$. The range can be extended to about $1.9 \pi$ by applying Euler's series transformation. The asymptotic expansion for large $\lambda$ is easily computed; a complete Maple program is supplied as a half-page table. The $N$-th order term is the product of a polynomial of degree $N-1$ with $\exp (-2 N \lambda)$ and thus the $N$ th order power-and-exponential series contains about $N^{2} / 2$ terms. The reward for this added complexity is that the series appears to converge over the entire range $\lambda \in[\pi / 2, \infty]$. The convergence rate is exponential except at the endpoint $\lambda=\pi / 2$.

The Never-Failing Newton's Initialization (NFNI) was the fruit of the trio of expansions. In this, it is a salutary reminder of the truism: Analysis is the father and mother of numerical analysis.

Acknowledgments: This work was supported by the National Science Foundation through grants OCE 0451951 and OCE 1059703. I thank the two reviewers for helpful comments.

\section{Appendix A. Computing the Roots of a Polynomial in Chebyshev Form}

The zeros of a truncated Chebyshev series on an interval $x \in[a, b]$ are most easily computed as the eigenvalues of a matrix as explained by the following [10].

Theorem 1 (Chebyshev Companion Matrix). Let $f_{N}(x)$ denote a polynomial of degree $N$ written in "Chebyshev form" on the interval $x \in[a, b]$ as

$$
f_{N}(x)=\sum_{j=0}^{N} a_{j} T_{j}\left(2 x-\frac{b+a}{b-a}\right)
$$

Then all roots of $f_{N}(x)$, both on and off the chosen expansion interval, $x \in[a, b]$, are eigenvalues of the $N \times N$ matrix $\overrightarrow{\vec{A}}$ whose elements are

$$
A_{j k}=\left\{\begin{array}{c}
\delta_{2, k}, \quad j=1, \quad k=1,2, \ldots, N \\
\frac{1}{2}\left\{\delta_{j, k+1}+\delta_{j, k-1}\right\}, \quad j=2, \ldots,(N-1), k=1,2, \ldots, N \\
(-1) \frac{a_{j-1}}{2 a_{N}}+(1 / 2) \delta_{k, N-1}, \quad j=N, k=1,2, \ldots, N
\end{array}\right.
$$

where $\delta_{j k}$ is the usual Kronecker delta function such that $\delta_{j k}=0$ if $j \neq k$ while $\delta_{j j}=1$ for all $j$. ([10].) The eigenvalues $n u_{j}$ of the matrix are the roots of $f(x[z])$ in $z$; these can be converted to $x$ via

$$
x=((b-a) / 2) z+(b+a) / 2
$$

(Independently proved by multiple authors as cataloged in [10].) 
For a quintic polynomial, for example,

$$
\left|\begin{array}{ccccc}
0 & 1 & 0 & 0 & 0 \\
(1 / 2) & 0 & (1 / 2) & 0 & 0 \\
0 & (1 / 2) & 0 & (1 / 2) & 0 \\
0 & 0 & (1 / 2) & 0 & (1 / 2) \\
(-1) \frac{a_{0}}{2 a_{5}} & (-1) \frac{a_{1}}{2 a_{5}} & (-1) \frac{a_{2}}{2 a_{5}} & (-1) \frac{a_{3}}{2 a_{5}}+(1 / 2) & (-1) \frac{a_{4}}{2 a_{5}}
\end{array}\right|
$$

Because the Chebyshev series is most accurate for $z \in[-1,1]$ or equivalently, $x \in[a, b]$, and the error grows exponentially fast off this interval, roots off this interval are suspect as approximations to the zeros of $f(x)$, the function approximated by $f_{N}$.

\section{References}

[1] M. Abramowitz And I. A. Stegun, Handbook of Mathematical Functions, Dover, New York, 1965.

[2] P. Amore, Alternative implementation of Pade approximants, Phys. Rev. D, 76 (2007), p. 076001.

[3] — Inveersiion of the perturbaion series, J. Phys. A Math. Theo., 41 (2008), p. 025201.

[4] P. Amore And F. M. Fernandez, Mathematical analysis of recent analytical approximations to the collapse of an empty spherical bubble, J. Chem. Phys., 138 (2013), p. 084511.

[5] J. P. BoyD, Orthogonal rational functions on a semi- infinite interval, J. Comput. Phys., 70 (1987), pp. 63-88.

[6] _ Chebyshev and Fourier Spectral Methods, Dover, New York, 2001. $680 \mathrm{pp}$.

[7] _ Solving Transcendental Equations: The Chebyshev Polynomial Proxy and Other Numerical Rootfinders, Perturbation Series and Oracles, SIAM, Philadelphia, 2010. 490 pp.

[8] — A proof, based on the Euler sum acceleration, of the recovery of an exponential (geometric) rate of convergence for the Fourier series of a function with Gibbs Phenomenon, in Spectral and Higher Order Methods for Partial Differential Equations: Proceedings of ICOSAHOM '09 Conference, June 22-26, Trondheim, Norway, J. S. Hesthaven and E. M. Ronquist, eds., no. 76 in Lecture Notes in Computational Science and Engineering, New York, 2011, Springer-Verlag, pp. 131-140.

[9] - Numerical, perturbative and Chebyshev inversion of the Incomplete Elliptic Integral of the Second Kind, Appl. Math. Comput., 218 (2012), pp. 7005-7013. 
[10] - Finding the zeros of a univariate equation: Proxy rootfinders, Chebyshev interpolation and the companion matrix, SIAM Rev., 55 (2013), pp. $375-396$.

[11] — Solving Transcendental Equations: The Chebyshev Polynomial Proxy and Other Numerical Rootfinders, Perturbation Series and Oracles, SIAM, Philadelphia, 2014. 460 pp.

[12] J. P. Boyd AND D. W. Moore, Summability methods for Hermite functions, Dyn. Atmos. Oceans, 10 (1986), pp. 51-62.

[13] R. BULIRSCH, Numerical computation of elliptic integrals and elliptic functions, Numer. Math., (1965), pp. 78-90.

[14] - Numerical computation of elliptic integrals and elliptic functions II, Numer. Math., 7 (1965), pp. 353-354.

[15] - Numerical computation of elliptic integrals and elliptic functions III, Numer. Math., 13 (1965), pp. 303-315.

[16] P. F. Byrd And M. D. Friedman, Handbook on Elliptic Integrals for Engineers and Physicists, Springer-Verlag, 2nd ed., 1971.

[17] B. C. Carlon, Computing elliptic integrals by duplication, Numer. Math., 33 (1979), pp. 1-16.

[18] — Numerical computation of real or complex elliptic integrals, Numer. Algorithms, 10 (1995), pp. 13-26.

[19] B. C. Carlson And E. M. Notis, Algorithm 577. Algorithms for incomplete elliptic integrals, ACM Trans. Math. Software, 7 (19), pp. 398-403.

[20] W. J. CoDy, Chebyshev approximations to the complete elliptic integrals $k$ and $e$, Math. Comput., 19 (1965), pp. 105-112.

[21] —, Chebyshev polynomial expansions of complete elliptic integrals, Math. Comput., 19 (1965), pp. 266-284.

[22] - Corrigenda: Chebyshev approximations to the complete elliptic integrals $k$ and $e$, Math. Comput., 20 (1966), pp. 207-207.

[23] A. R. DiDonato And A. V. Hershey, New formulas for computing incomplete elliptic integrals of the first and second kind, J. Assoc. Comput. Mach., 6 (1959), pp. 515-526.

[24] T. Fukushima, Precise and fast computation of a general incomplete elliptic integral of second kind by half and double argument transformations, J. Comp. Appl. Math., 235 (2011), pp. 4140-4148.

[25] — Series expansion of symmetric elliptic integrals, Math. Comp., (2011), p. in press. 
[26] - Numerical computation of inverse complete elliptic integrals of first and second kinds, J. Comput. Appl. Math., 249 (2013), pp. 37-50.

[27] - Numerical inversion of a general incomplete elliptic integral, J. Comput. Appl. Math., 237 (2013), pp. 43-61.

[28] G. E. Lee-Whiting, Formulas for computing incomplete elliptic integrals of the first and second kinds, J. Assoc. Comput. Mach., 10 (1963), pp. 126130 .

[29] P. M. Morse And H. Feshbach, Methods of Theoretical Physics, (two volumes), McGraw-Hill, New York, 1953.

[30] K. Oldham and J. M. aAnd Jerome Spanier, An Atlas of Functions: with Equator, the Atlas Function Calculator, Springer, New York, 2d ed., 2008. 750 pp.

[31] F. W. J. Olver, D. W. Lozier, R. F. Boisvert, and C. W. Clark, eds., NIST Handbook of Mathematical Functions, Cambridge University Press, New York, 2010.

[32] C. J. Pearce, Transformation methods in the analysis of series for critical properties, Adv. Phys., 27 (1978), pp. 89-148.

[33] H. VAN DE VEL, On the series expansion method for computing incomplete elliptic integrals of the first and second kinds, Math. Comp., 23 (1969), pp. 61-69. 


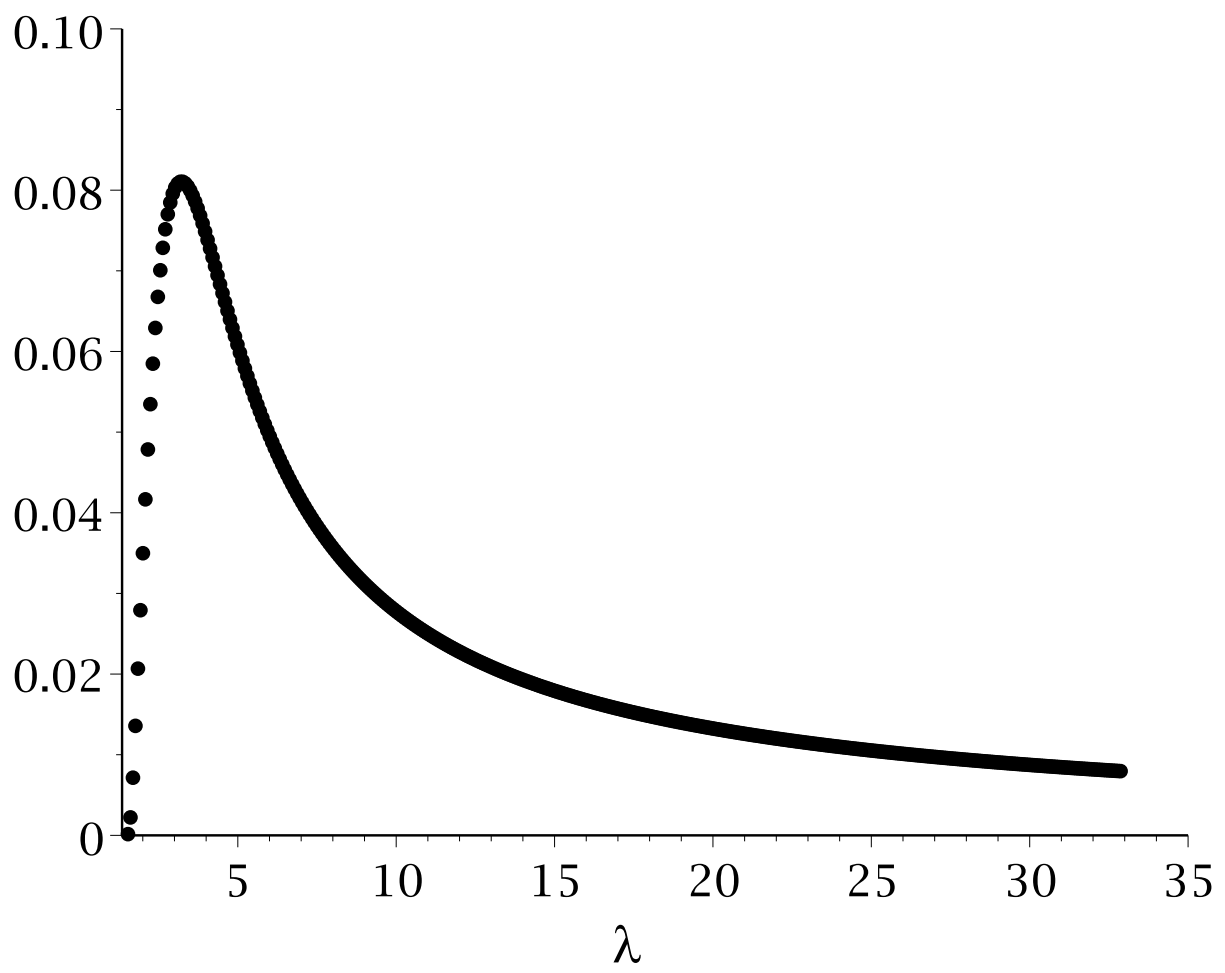

Figure 3: Relative initial error in $x$ for the Never-Failing Newton Initialization, $x \approx$ $\exp (-\bar{\lambda}[2.54+2.96 \bar{\lambda}] /[1+1.48 \bar{\lambda}]$. Very high precision arithmetic was used for $\lambda>10$ because $x$ is very, very tiny; we recommend the asymptotic approximation in place of Newton's iteration for large $\lambda$. 


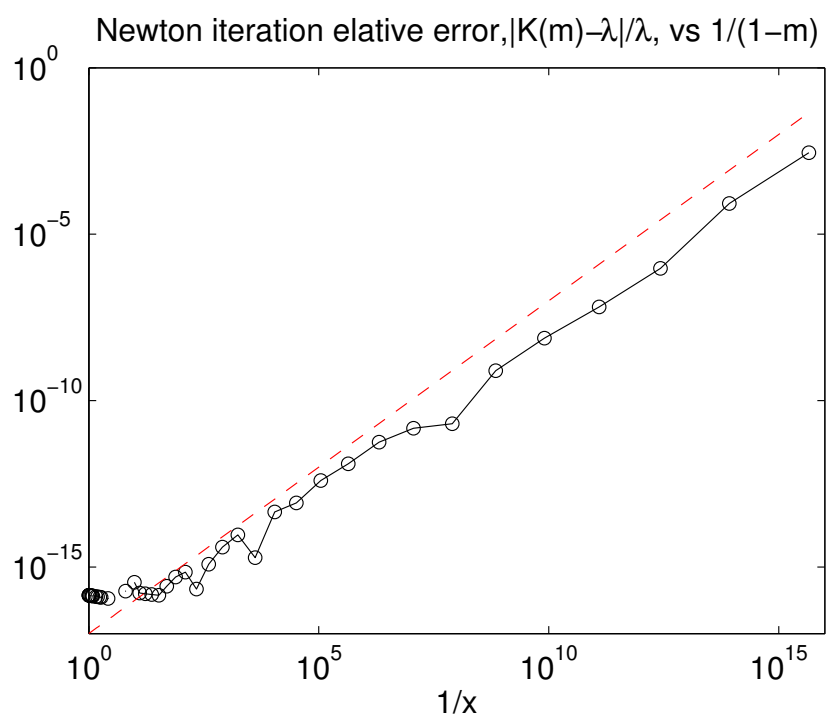

Figure 4: When the elliptic modulus $m$ is very close to its limiting value of one, which is equivalent to $x<<1$ where $x \equiv 1-m$, the relative error afte Newton's iteration rises roughly linearly with $1 / x$. The red dashed line is $10^{-17}$, which is an upper bound on the error. 